\title{
Origen y destino de recursos del presupuesto de los GADs Municipales: estudio del Cantón Morona
}

\section{Origin and destination of resources of the budget of the Municipal GADs: study of the Canton Morona}

\author{
Ayala Pasquel, Sara Nathaly ${ }^{1 *}$, Zaruma Ávila, María Ester ${ }^{1}$ y Barragán Sánchez, Hilda María ${ }^{1}$ \\ ${ }^{1}$ Universidad Católica de Cuenca, Sede Macas, Ecuador \\ *sayalap@ucacue.edu.ec
}

\begin{abstract}
Resumen
Los Gobiernos Autónomos Descentralizados Municipales se crearon con la finalidad de poseer autonomía para planificar y ejecutar proyectos de inversión, a través de la obtención y generación de distintas fuentes de ingresos que solventan la ejecución de los mismos de acuerdo a la normativa vigente en Ecuador. La investigación brinda información sobre el origen y los tipos de ingresos que adquieren los GAD Municipales y el procedimiento para la obtención, así como, la aplicabilidad y utilización, tomando como base el estudio de la normativa legal vigente, el Código Orgánico de Organización Territorial, el Código Orgánico de Planificación y Finanzas Públicas, entre otros, y la practicidad que realizan los municipios basados en la experiencia y buena gobernabilidad. La metodología utilizada fue el estudio de caso, considerando como objeto de disertación al GAD Municipal del Cantón Morona, analizando la aproximación a las normas legales e investigando las características básicas del caso de estudio, se examinan las fases de elaboración del presupuesto, lo que permite sustentar los datos recogidos en la investigación. Se ha optado por un estudio de caso en vista que las municipalidades del Ecuador se desenvuelven bajo normas similares con algunos casos de excepciones. Uno de los principales resultados indica que los gastos de inversión, son el tipo de gastos más importante dentro de los GADS ya que en los mismos se refleja el total de recursos invertidos en la ejecución de programas y proyectos en beneficio de la población. Entre otros aspectos se concluye que es fundamental que los contribuyentes tomen conciencia de la importancia de cumplir con las obligaciones tributarias ante la Municipalidad, de tal manera que la entidad pueda gestionar los fondos recaudados por los habitantes para realizar obras que beneficien el crecimiento y desarrollo su jurisdicción.
\end{abstract}

Palabras clave: Gobierno Autónomo Descentralizado, presupuesto, origen, destino. .

\begin{abstract}
The Decentralized Municipal Autonomous Governments were created with the purpose of having autonomy to plan and execute investment projects, through the obtaining and generation of different income sources that solve the execution of the same according to the current regulations in Ecuador. The research provides information on the origin and types of income that the Municipal GADs acquire and the procedure for obtaining it, as well as the applicability and use, based on the study of current legal regulations, the Organic Code of Territorial Organization, the Organic Code of Planning and Public Finances, among others, and the practicality that municipalities perform based on experience and good governance. The methodology used was the case study, considering as an object of dissertation to the GAD Municipal del Cantón Morona, analyzing the approximation to the legal norms and investigating the basic characteristics of the case of study, the phases of preparation of the budget are examined, which allows sustain the data collected in the investigation. A case study has been chosen in view of the fact that the municipalities of Ecuador operate under similar rules with some cases of exceptions. One of the main results indicates that investment expenses are the most important type of expenditure within the GADS since they reflect the total resources invested in the execution of programs and projects for the benefit of the population. Among other aspects, it is concluded that it is essential that taxpayers become aware of the importance of complying with tax obligations before the Municipality, in such a way that the entity can manage the funds collected by the inhabitants to carry out works that benefit the growth and development of their jurisdiction.
\end{abstract}

Key words: Decentralized Autonomous, Government, budget, origin, destination. .

\section{Introducción}

Los Municipios se originaron en Roma y fueron creados con la finalidad de gobernar a la población a través de las propias leyes implementadas por los gobernantes.
En América, las primeras ciudades fundadas formaban un Municipio, cada una representada por su cabildo que a su vez dependía del gobernador o Alcalde Mayor. Los Cabildos tenían potestad en el campo legislativo y se encargaban 
del manejo de asuntos importantes de la comunidad local. (Ojeda, 2007).

En Ecuador, durante la Constitución de 1850 se crean municipalidades en cada cabecera cantonal y en 1861 se señala la creación de municipios en las Provincias, Cantones y Parroquias. Durante la Constitución de 1946 se reconoce a los municipios como entidades autónomas, con potestad para dictar ordenanzas, crear, modificar y suprimir tasas de contribuciones de mejoras. (Cabrera, 2014). En 1971 se publica la Ley Orgánica del Régimen Municipal (LOREM), mediante Suplemento del Registro Oficial 331 del 15 de octubre de 1971 ("Gobierno Parroquial Rural La Merced", 1971), bajo la cual se regían las municipalidades del Ecuador; ya en el año 2008 se reformó, cuando se dio el cambio en todo el marco jurídico a través de la constituyente en Montecristi, y se publica el nuevo sustento legal denominado Código Orgánico de Organización Territorial (COOTAD), bajo el cual se establecen actualmente las funciones y competencias para las municipalidades y se desarrolla un modelo de descentralización obligatoria y progresiva a través del Sistema Nacional de Competencias, la institucionalidad responsable de su administración, las fuentes de financiamiento y la definición de políticas y mecanismos para compensar los desequilibrios en el desarrollo territorial. (Ministerio de Coordinación de la Política y Gobiernos Autónomos Descentralizados, 2010). A partir de esta Normativa toman el nuevo nombre de Gobiernos Autónomos Descentralizados (GAD)

Las municipalidades del Ecuador tienen la función de desarrollar proyectos y obras en beneficio de la población observando los principios comunes que rigen a los GADs municipales del Ecuador, de acuerdo al Código Orgánico de Planificación Y Finanzas Públicas en el art. 5 indica que debe existir planificación que cuente con las siguientes fases: La programación, formulación, aprobación, asignación, ejecución, seguimiento y evaluación del Presupuesto General del Estado, los mismos que estarán bajo los lineamientos de la planificación del desarrollo de todos los niveles de gobierno, en observancia a lo dispuesto en los artículos 280 y 293 de la Constitución de la República (COPFP, 2010)

\subsection{Ingresos percibidos por las Municipalidades de acuerdo a la Normativa y sus competencias.}

Con la finalidad de establecer los ingresos percibidos por las municipalidades en el Ecuador, el Código Orgánico de Planificación y Finanzas Publicas (COPFP) en el art. 78 los clasifica en permanentes y no permanentes. Este Código determina que los ingresos permanentes son los provenientes de:

Recursos públicos que el Estado a través de sus entidades, instituciones y organismos públicos reciben de manera continua, periódica y previsible. La generación de ingresos permanentes no ocasiona la disminución de la riqueza nacional por cuanto no provienen de enajenación, degradación o venta de activos públicos de ningún tipo o del endeudamiento público. Mientras tanto, determina que los Ingresos no-permanentes son los ingresos de recursos públicos que el Estado a través de sus entidades, instituciones y organismos, reciben de manera temporal, por una situación específica, excepcional o extraordinaria. La generación de ingresos no permanentes puede ocasionar disminución de la riqueza nacional. Por ello, los ingresos no permanentes pueden provenir, entre otros, de la venta de activos públicos o del endeudamiento público.(COPFP, 2010)

El código Orgánico de Planificación y Finanzas Públicas señala que a los Gobiernos Municipales Descentralizados en concordancia con la Constitución de la República se le podrán asignar nuevas competencias para lo cual se debe transferir recursos o contarán con la autonomía de gestión de los mismos, tal es el caso, que conforme a la Resolución $\mathrm{N}^{\circ} 006$ del Concejo Nacional de Competencias, con el objetivo de consolidar un nuevo régimen de desarrollo, centrado en el buen vivir, a través de un modelo de autonomías que aporten en la construcción de un proceso justo y equilibrado del territorio nacional, en mayo del 2015 la Agencia Nacional de tránsito (ANT), según mandato constitucional con la finalidad de mejorar la calidad del servicio para el usuario, entregó las competencias en materia de transporte terrestre, tránsito y seguridad vial a los GADs del país. (Agencia Nacional de Tránsito, 2014). Desde ese instante forman parte de los presupuestos municipales, los ingresos por ese concepto.

Los gobiernos Autónomos Municipales del Ecuador gozan de total autonomía que ninguna instancia del Estado podrá interferir en sus gestión, como se señala en el COOTAD, el art. 6 que menciona: "ninguna función del Estado ni autoridad extraña podrá interferir en la autonomía política, administrativa y financiera propia de los Gobiernos Autónomos Descentralizados, salvo lo prescrito por la Constitución y las leyes de la República". (Código Orgánico Organización Territorial, Autonomía y Descentralización, 2010). Uno de estos casos se estipula en el mismo código en el art. 170 donde menciona que estas entidades autónomas pueden establecer subsidios en el cobro por la prestación de los servicios básicos aplicando un sistema de subsidios solidarios cruzados entre los sectores de mayores y menores ingresos. (Código Orgánico Organización Territorial, Autonomía y Descentralización, 2010), lo que se puede concluir que cada municipalidad podrá tener ingresos de acuerdo a la calidad de vida de sus habitantes, tal es así que no se podría comparar una municipalidad en lugares que tienen poco acceso a servicios básicos y su población es de un nivel económico limitado.

Con el propósito de realizar la gestión pública, la COOTAD, en su artículo 171, señala la potestad que tiene de percibir, como fuente, los tipos de recursos financieros de los Gobiernos Autónomos Descentralizados y señala que serán los siguientes: 
Tabla 1

Tipos de Recursos Financieros de los GADs

\begin{tabular}{|ll|}
\hline \multicolumn{2}{|c|}{ Ingresos de los GADS } \\
\hline - & Ingresos propios de la gestión \\
\hline - & Transferencias del presupuesto General del Estado \\
\hline - & Ingresos por transferencias, legados o donaciones \\
\hline - & Participación en las rentas de la explotación o \\
& industrialización de recursos naturales no renovables \\
\hline$\bullet$ & Recursos provenientes de financiamiento. \\
\hline
\end{tabular}

Fuente: (Código Orgánico Organización Territorial, Autonomía y Descentralización, 2010)

1.2 Gastos generados por las Municipalidades de acuerdo a la Normativa y sus competencias

Dentro de las competencias asignadas a los GADs está la de satisfacer las necesidades del Cantón al que se deben, a través de la ejecución de obras públicas, para ello deben existir distintos tipos de gastos como son:

- Gastos Corrientes: son gastos operativos para que exista en ente gubernamental que realice obra, dentro de ello esta los gastos en personal, bienes y servicios de consumo, gastos financieros, otros gastos corrientes y transferencias y donaciones corrientes.

- Gastos de Inversión: gastos en personal para inversión, bienes y servicios para inversión, obras públicas, otros gastos de inversión, transferencias y donaciones para inversión.

- Gastos de Capital: gastos destinados a la compra de activos fijos que mantiene la institución, bienes de larga duración.

- Aplicación del Financiamiento: Amortización de la deuda pública, pasivo circulante.

De la misma forma que los ingresos, los egresos fiscales también se clasifican en en egresos permanentes y no permanentes, y éstos podrán subclasificarse en otras categorías con fines de análisis, organización presupuestaria y estadística.

Egresos permanentes: egresos de recursos públicos que el Estado a través de sus entidades, instituciones y organismos, efectúan con carácter operativo que requieren repetición permanente y permiten la provisión continua de bienes y servicios públicos a la sociedad, y estos no podrán ser financiados con ingresos no permanentes, de acuerdo la normativa vigente. Los egresos permanentes no generan directamente acumulación de capital o activos públicos, se realizan de manera temporal, para actividades de situación específica, excepcional o extraordinaria que no requiere repetición permanente.

Los egresos permanentes se podrán financiar con ingresos no permanentes en las situaciones excepcionales que prevé la Constitución de la República, para salud, educación y justicia; previa calificación de la situación excepcional, realizada por el Presidente de la República. (Código Orgánico Organización Territorial, Autonomía y Descentralización, 2010).

Para determinar la aplicabilidad y cumplimiento de la Normativa antes expuesta se analiza en esta investigación el origen, procedimiento y uso de los recursos que forman parte de los presupuestos de los GADs Municipales tomando como caso de estudio el GAD Municipal con el nombre del mismo Cantón ubicado en la Provincia de Morona Santiago, Cantón Morona, cabecera cantonal Macas, que fue creado por disposición Constitucional y expuesta mediante decreto publicado en el Registro Oficial N. 172 de 25 de marzo de 1897. (Gobierno Municipal del Cantón Morona, s.f.)

Este GAD, está encargado de planificar, implementar e impulsar proyectos y servicios de calidad con la finalidad de satisfacer necesidades colectivas, a su vez asegurando el desarrollo sostenible, equitativo, competitivo, a través de la participación directa de los actores sociales, en un marco de ética y transparencia institucional, optimizando los recursos disponibles. (Gobierno Municipal del Cantón Morona, s.f.)

\section{Metodología}

En la presente investigación se utilizó la metodología estudio de caso con un tipo de investigación exploratoria, descriptiva y de análisis documental. La recolección de la información se obtuvo a través de entrevistas y cuestionarios realizados a los responsables del área financiera y de presupuestos en el Gobierno Autónomo Descentralizado del Cantón Morona, aplicando la técnica exploratoria, a través de la cual se obtuvo información relevante respecto al tema de estudio y permitió describir la aplicación de los procedimientos observados y establecer un análisis pormenorizado del mismo. Para el análisis documental se realizó revisión de reglamentos, normativa, ordenanzas aplicadas a los Municipios, información sujeta a análisis de la teoría respecto a la aplicación de prácticas, así como también tesis similares.

\section{Resultados y discusión}

En los resultados obtenidos después de realizar la investigación en el GAD Municipal del Cantón Morona con respecto origen, procedimiento y destino de los recursos que forman parte de los presupuestos de los GADs Municipales, se determina lo siguiente: 
3.1 Origen de los Ingresos que mantiene el GAD del Cantón Morona

Tabla 2

Ingresos Corrientes del GAD del Cantón Morona

\begin{tabular}{|ll|}
\hline \multicolumn{2}{|c|}{ INGRESOS CORRIENTES } \\
\hline - & Impuestos (11) \\
\hline - & Tasas y Contribuciones (13) \\
\hline - & Venta de Bienes y Servicios (14) \\
\hline - & Rentas de Inversiones y Multas (17) \\
\hline - & Transferencias y Donaciones Corrientes (18) \\
\hline - Otros Ingresos (19) \\
\hline
\end{tabular}

Fuente: Base de Datos del Presupuesto del GAD Municipal del Cantón Morona

De acuerdo a la información obtenida se determina que actualmente la Municipalidad mantiene ingresos corrientes que forman parte de los ingresos propios, Transferencias del Presupuesto General del Estado, e ingresos por transferencias legados y donaciones, estos ingresos depende de acuerdo a la normativa la recaudación que obtienen de acuerdo a las ordenanzas y Reglamentos Municipales que les faculta según el COOTAD a recaudar recursos por cuenta propia, además existe también recursos que el Estado le asigna por recursos permanentes, así como también de acuerdo a la normativa legal vigente pueden participar de los recursos que se genera por conceptos de transferencia de legados y donaciones.

Parte de los Ingresos Corrientes que son de gestión propia de las Municipalidad están dados por el cobro de los predios urbanos y rústicos, patentes por la funcionalidad de negocios en el Cantón, ingresos generados a través de ordenanzas por las mismas obras construidas por el $\mathrm{Mu}-$ nicipio, trámites vehiculares y terrenos, servicios básicos, áreas verdes, asfaltos, ingresos permanentes del $30 \%$ del Presupuesto General del Estado de cada año, entre otros.

Tabla 3

Ingresos de Capital del GAD del Cantón Morona

\begin{tabular}{l} 
INGRESOS DE CAPITAL \\
\hline - Venta de Activos no Financieros \\
\hline - Transferencias y Donaciones de Capital E Inversión \\
\hline $\begin{array}{l}\text { Fuente: Base de Datos del Presupuesto del GAD Municipal del } \\
\text { Cantón Morona }\end{array}$
\end{tabular}

Los Ingresos de Capital provienen el $70 \%$ de ingresos recibidos del Presupuesto General del Estado, corres- pondiente al año en curso, ingresos de Entidades Financieras Públicas para la ejecución de obras en proceso o por realizarse, ingresos por la venta de bienes muebles e inmuebles e ingresos de acuerdo la ley 10 del fondo para el Ecodesarrollo Regional Amazónico (ECORAE), que fue creada para recaudar ingresos por impuesto que equivale a un dólar por cada barril de petróleo extraído de la Región Amazónica, esta ley menciona que del fondo recaudado el $58 \%$ será destinado para los GAD municipales de la Amazonía; así como también, reciben ingresos del $5 \%$ por la facturación total en la venta de energía eléctrica de la central hidroeléctrica de Paute, según lo establece la Ley 047.

Tabla 4

Ingresos de Financiamiento del GAD del Cantón Morona

\begin{tabular}{|ll|}
\hline \multicolumn{1}{|c|}{ INGRESOS DE FINANCIAMIENTO } \\
\hline - & Financiamiento Público \\
\hline - & Saldos Disponibles \\
\hline - & Cuentas Pendientes por Cobrar \\
\hline
\end{tabular}

Fuente: Base de Datos del Presupuesto del GAD Municipal del Cantón Morona

Finalmente los Ingresos de Financiamiento son aquellos ingresos que la municipalidad obtiene de las Entidades Financieras Públicas para la construcción de obras planificadas por el GAD Municipal del Cantón Morona, reciben únicamente fondos del Banco de Desarrollo.

En la siguiente tabla 5 se presenta un resumen de los ingresos presupuestados por el GAD Municipal del Cantón Morona para el año 2017.

En el detalle mencionado se puede observar que de acuerdo al presupuesto planificado de la Municipalidad se espera recaudar durante el año 2017 la cantidad de $\$ 38^{\prime} 339.383 .60$, respecto a ingresos corrientes, de capital y financiamiento que actualmente dispone la entidad.

De la totalidad de ingresos presupuestados para el año 2017 , el $14.96 \%$ corresponde a los ingresos corrientes presupuestados que obtendrá la municipalidad, los ingresos de capital representan el $55 \%$ y con el $30 \%$ están representados los ingresos por financiamiento. Cabe mencionar que los ingresos de capital cuentan con el mayor porcentaje de ingresos presupuestados para el año 2017, debido a que en este rubro constan las transferencias recibidas del Presupuesto General del Estado como ingresos no permanentes destinadas a la ejecución y utilización en ejecución de obra pública; también esta las transferencias de entidades financieras públicas para que el Municipio pueda desarrollará las actividades de construcción de obras en el Cantón.

\subsection{Destino de los Recursos del GAD del Cantón Morona}

La Municipalidad a más de sus ingresos presupuestados establece también los diferentes tipos de gastos que 
Tabla 5

Cédula Presupuestaria de Ingresos 2017 del GAD del Cantón Morona

\begin{tabular}{|c|c|c|}
\hline \multicolumn{3}{|c|}{ CÉDULA PRESUPUESTARIA DE INGRESOS } \\
\hline CÓDIGO & DENOMINACIÓN & VALOR INICIAL \\
\hline 10.00 .00 .00 & INGRESOS CORRIENTES & $5.734 .631,25$ \\
\hline 11.00 .00 .00 & Impuestos & $1.143 .600,00$ \\
\hline 13.00 .00 .00 & Tasas y Contribuciones & $1.269 .444,04$ \\
\hline 14.00 .00 .00 & Venta de Bienes y Servicios & $382.300,00$ \\
\hline 17.00 .00 .00 & Rentas de Inversiones y Multas & $398.400,00$ \\
\hline 18.00 .00 .00 & Transferencias y Donaciones Corrientes & $1.940 .787,21$ \\
\hline 19.00 .00 .00 & Otros Ingresos & $600.100,00$ \\
\hline 20.00 .00 .00 & INGRESOS DE CAPITAL & $21.078 .880,14$ \\
\hline 24.00 .00 .00 & Venta de Activos no Financieros & $8.100,00$ \\
\hline 28.00 .00 .00 & Transferencias y Donaciones de Capital e Inversión & $21.070 .780,14$ \\
\hline 30.00 .00 .00 & INGRESOS DE FINANCIAMIENTO & $11.525 .872,21$ \\
\hline 36.00 .00 .00 & Financiamiento Público & $5.362 .484,47$ \\
\hline 37.00 .00 .00 & Saldos Disponibles & $1.600 .000,00$ \\
\hline 38.00 .00 .00 & Cuentas Pendientes por Cobrar & $4.563 .387,74$ \\
\hline & TOTALES & $38.339 .383,60$ \\
\hline
\end{tabular}

Fuente: Base de Datos del Presupuesto del GAD Municipal del

Cantón Morona

incurrirán en el desarrollo de sus funciones, a continuación, se presenta los gastos presupuestados para el año 2017 que tendrá el GAD Municipal del Cantón (Ver tabla 6 )

La totalidad de gastos durante el año 2017 que proyecta utilizar la entidad es de \$38’339.383,60. De acuerdo a la totalidad de gastos indicados, se prevé que se utilizará el $10 \%$ en gastos corrientes de la Municipalidad, que servirán para solventar gastos del personal administrativo, beneficios sociales, servicios básicos, aportes fiscales, gastos financieros, transferencias corrientes, entre otros.

De la misma manera el $88 \%$ representa los gastos de inversión, este tipo de gastos es el más importante dentro de los GADS ya que este rubro refleja el total de recursos invertidos en la ejecución de programas y proyectos en beneficio de población, en los cuales conforman los gastos de personal encargados de realizar las obras, bienes y servicios para inversión, obras públicas y demás gastos que estén relacionados a la inversión que realizará el Municipio, primordialmente está enfocada realización de mejoras viales, servicios de agua potable y demás, mismos que contribuyen con el mejoramiento de la calidad de vida y buen vivir de los ciudadanos del Cantón.

Con igual representación del $1 \%$ corresponden a los gastos de capital y aplicación del financiamiento, en donde 
Tabla 6

Cédula Presupuestaria de Gastos 2017 del GAD del Cantón Morona

\begin{tabular}{|c|c|c|}
\hline \multicolumn{3}{|c|}{ CÉDULA PRESUPUESTARIA DE GASTOS } \\
\hline CÓDIGO & DENOMINACIÓN & VALOR INICIAL \\
\hline 0.50 .00 .00 .000 & GASTOS CORRIENTES & $3.762 .784,85$ \\
\hline 00.51 .00 .00 .000 & Gastos en Personal & $2.676 .668,31$ \\
\hline 00.53 .00 .00 .000 & Bienes y Servicios de Consumo & $570.309,00$ \\
\hline 00.56 .00 .00 .000 & Gastos Financieros & $396.807,54$ \\
\hline 00.57 .00 .00 .000 & Otros Gastos Corrientes & $29.000,00$ \\
\hline 00.58 .00 .00 .000 & $\begin{array}{l}\text { Transferencias y Donaciones } \\
\text { Corrientes }\end{array}$ & $90.000,00$ \\
\hline 00.70.00.00.000 & GASTOS DE INVERSIÓN & 33.732.278,41 \\
\hline 00.71 .00 .00 .000 & Gastos en Personal para Inversión & $3.943 .533,03$ \\
\hline 00.73 .00 .00 .000 & Bienes y Servicios para Inversión & $5.104 .523,22$ \\
\hline 00.75 .00 .00 .000 & Obras Públicas & $24.466 .290,54$ \\
\hline 00.77 .00 .00 .000 & Otros Gastos de Inversión & $97.931,62$ \\
\hline 00.78 .00 .00 .000 & $\begin{array}{l}\text { Transferencias y Donaciones para } \\
\text { Inversión }\end{array}$ & $120.000,00$ \\
\hline 00.80.00.00.000 & GASTOS DE CAPITAL & $436.868,00$ \\
\hline 00.84 .00 .00 .000 & Bienes de Larga Duración & $436.868,00$ \\
\hline 00.90.00.00.000 & $\begin{array}{l}\text { APLICACIÓN DEL } \\
\text { FINANCIAMIENTO }\end{array}$ & $407.452,34$ \\
\hline 00.96 .00 .00 .000 & Amortización de la Deuda Pública & $402.452,34$ \\
\hline 00.97 .00 .00 .000 & Pasivo Circulante & $5.000,00$ \\
\hline & TOTALES & $38.339 .383,60$ \\
\hline
\end{tabular}

Fuente: Base de Datos del Presupuesto del GAD Municipal del Cantón Morona

los valores monetarios asignados a los gastos de capital servirán para realizar adquisiciones de bienes de larga duración como las maquinarias para la ejecución de las obras, y en la aplicación del financiamiento corresponde para realizar pagos de deuda pública y para los pagos de deudas pendientes de pago del ejercicio fiscal anterior y del actual. Los gastos de capital nos indican cual es la cantidad porcentual y relativa que la institución ha invertido en la compra de activos fijos que ayudan y cooperan en la ejecución de los proyectos y programas previamente planificados dentro del Plan de Desarrollo cantonal.

\section{Conclusiones}

Al concluir la investigación y con los resultados obtenidos se determina que el GAD Municipal del Cantón Morona para realizar sus funciones se sustenta de los ingresos obtenidos por su gestión propia, por las transferencias recibidas del Presupuesto General del Estado y Entidades Financieras Públicas, que pertenecen a ingresos permanentes y no permanentes, mediante financiamiento público. La obtención de estos fondos ayuda a que la entidad pueda realizar proyectos, entre los más relevantes la construcción de obras, proyectos de mejora y la prestación de servicios públicos, favoreciendo de tal manera el mejoramiento de la calidad de vida de los habitantes.

La administración Municipal del Cantón Morona, previa planificación, a través de una forma participativa visitan a las comunidades conformadas en el Cantón para conocer las necesidades requeridas por los habitantes, elabora y aprueba el presupuesto, el mismo que le sirve como herramienta de gestión para el cumplimiento de obras y servicios demandados por la sociedad, que contribuyan al desarrollo de su población.

Es fundamental que los contribuyentes tomen conciencia de la importancia de cumplir con las obligaciones tributarias ante la Municipalidad, de tal manera que la entidad pueda gestionar los fondos recaudados por los habitantes para realizar obras que beneficien el crecimiento de su jurisdicción.

Es necesario realizar una campaña de socialización a los ecuatorianos acerca de los procesos, y funciones que 
realizan cada uno de los GADs Municipales en el Ecuador, con la finalidad de contribuir a que los ciudadanos tomen conciencia y reflexión total de la importancia que tiene para los Municipios, la contribución de obligaciones tributarias Municipales, ya que a través de los valores recaudados, estas entidades Municipales podrán ejercer una de sus funciones principales, que es la inversión para la construcción de obras o proyectos de mejora de los principales servicios públicos que necesita los seres humanos para tener una mejor calidad de vida.

\section{Referencias Bibliográficas}

Agencia nacional de tránsito. (2014). Descargado de http: //www.ant.gob.ec

Alexandra, J., y Johana, M. http://repositorio.utmachala.edu.ec.

Cabrera, E. (2014). Universidad andina simón bolívar.

Código orgánico organización territorial, autonomía y descentralización. (2010).

COPFP, C. O. d. P. y. F. P. (2010). COPFP, codigo orgánico de planificación y finanzas públicas. Corporacion de Estudios y Publicaciones.

Gobierno municipal del cantón morona. (s.f.).

Gobierno parroquial rural la merced. (1971).

http://www.finanzas.gob.ec. (2017).

http://www.oas.org. (2010).

Juana, B. (2016). Universidad de guayaquil.

Ojeda, J. (2007a). http://repositorionew.uasb.edu.ec.

Ojeda, J. (2007b). Universidad andina simón bolívar.

Recibido: 25 de mayo de 2017

Aceptado: 12 de octubre de 2017 
